\title{
Prologue to Linear Algebra
}

\author{
Y.Saroja, P.Shirisha, K.Kiran Kumar
}

\begin{abstract}
In this we're demonstrating an analyze on the instantaneous polynomial math and shape in huge variety juggling. right now component based totally totally math is the piece of range juggling required with the exam of vectors, vector domain names (in like manner encouraged as straight away zones), instantly maps (moreover insinuated as right away systems), and systems of heterosexual conditions. Vector districts are a critical check in current math; on this manner, immediately factor primarily based math may be very well applied in every applied polynomial math and critical assessment. Direct polynomial math likewise has a strong occasion in logical geometry and it is summed up in government rule. It has great packages inside the home advanced sciences and the humanistic structures, considering nonlinear fashions can continuously be approximated via the usage of direct ones.
\end{abstract}

\section{INTRODUCTION}

Instantly polynomial math had its beginnings within the have a take a look at vectors in Cartesian 2-spot and 3-area. A vector, real proper here, is an organized line parcel, portrayed via the two its centrality, addressed thru technique for range, and its way. Vectors may be used to set up terrific components which encompass forces, and they may be conveyed to every different and enlivened with scalars, ultimately molding the precept instance of a veritable vector region. contemporary instantly element based math has been attracted out to hold up beneath as a vital want zones of optionally available or endless period.

A vector area of estimation $n$ is known as a n-region. maximum of the accommodating effects from 2 -and 3 -space may be postponed to these better dimensional regions. irrespective of the manner that humans can not successfully envision vectors in $\mathrm{n}$-place, such vectors or $\mathrm{n}$-tuples are substantial in addressing surenesses. for the reason that vectors, as n-tuples, are mentioned publications of movement of $\mathrm{n}$ elements, it is feasible to consolidate and direct research essentially on this framework. for instance, in monetary viewpoints, you could make and use, say, eightdimensional vectors or 8-tuples to cope with the Gross throughout the us of a made of eight countries. You in all likelihood can pick out to expose the GNP of 8 all inclusive territories for a selected 12 months, wherein the nations' shape is amazing, as a case, (the us, united kingdom, France, Germany, Spain, India, Japan, Australia), thru using a vector (v1, v2, v3, v4, v5, v6, v7, v8) wherein every the usa's GNP is in its character restrict. A vector area (or direct territory), as a factor of reality hypothetical concept round which theories are illustrated, is a chunk of low cost variable primarily based math, and is all round joined into this case. a couple setting occasions of this are the association of

Revised Manuscript Received on April 12, 2019.

Y.Saroja, MallareddyCollege of Engineering for Women, Hyderabad. Telangana, India.

P.Shirisha, MallareddyCollege of Engineering for Women, Hyderabad.Telangana, India.

K.Kiran Kumar, PVP Siddhartha Institute of Technology, Kanuru, Vijayawada.A.P, India. invertible direct maps or grids, and the ring of straight maps of a vector vicinity. Direct polynomial math moreover plays a essential factor in assessment, considerably, inside the depiction of higher solicitation subordinates in vector appraisal and the take a look at tensor item and pivoting maps. on this edited version placing, the scalars with which a part of a vector place can be advanced want no longer be numbers. The high-quality need is that the scalars structure a logical structure, known as a place. In ventures, this trouble is regularly the hover of right numbers or the spot of complicated numbers. Direct maps take segments from a without delay district to every other (or to itself), in a manner this is superb with the improvement and scalar increment given on the vector vicinity(s). The direction of movement of each and each such adjustment is itself a vector place. In case a cause at the back of a vector spot is constant, each right away alternate can be addressed via a desk of numbers known as a network. The valid exam of the homes of and computations acting on circulate sections, together with determinants and eigenvectors, is contemplated to be a bit of direct aspect based totally math. you could say really truly that the immediately burdens of wide variety juggling - folks who display linearity of their lead - are the ones biggest conceivable to be lit up. for instance differential math finishes a wonderful oversee immediately gauge to limits. The qualification from nonlinear bothers may be astoundingly crucial in walking out. the overall process for finding a right away technique to have an eye an trouble, supplying this with reference to instantly aspect based totally math, and solving it, if need be with the benefit of matrix exams, is a standout amongst the maximum first-rate conventionally fabric in calculating.

\section{DIRECT ALGEBRA WHAT IS LINEAR ALGEBRA?}

Straight Algebra is the branch of math proposed for managing structures of direct conditions with a limited amount of inquiries. Remarkably, one should want to find out answers to the subsequent inquiries:

- Characterization of pastime plans: Are there solutions for a given device of hetero situations? how many solutions are there?

Coming across sport plans: How does the sport plan set appearance? What are the publications of movement? immediately Algebra is a unique idea concerning the sport plans of systems of direct situations.

Occasion 2.1. Empower us to take the following contraption of heterosexual situations in the inquiries $\mathrm{x} 1$ and

$\mathrm{x} 2$ :

$$
\left.\begin{array}{r}
2 x_{1}+x_{2}=0 \\
x_{1}-x_{2}=1
\end{array}\right\} .
$$


This system has a unique solution for $x_{1}, x_{2} \in \mathbb{R}$, namely $x_{1}=\frac{1}{3}$ and $x_{2}=-\frac{2}{3}$.

The solution can be located in numerous one of a kind techniques. One technique is to first clear up for one of the unknowns in one of the equations and then to replacement the give up result into the opposite equation. here, for instance, we might clear up to obtain

$$
\mathrm{X} 1=1+\mathrm{x} 2
$$

From the second one equation. Then, substituting this in area of $x 1$ within the first equation, we have $2(1+x 2)+x 2$ $=$ zero:From this, $x_{2}=-2 / 3$. Then, by further substitution,

$$
x_{1}=1+\left(-\frac{2}{3}\right)=\frac{1}{3} .
$$

Alternatively, we can take a more systematic approach in eliminating variables. Here, for example, we can subtract 2 times the second equation from the first equation in order to obtain $3 \times 2=-2$. It is then immediate that $\times 2=-2 / 3$ and, by substituting this value for $\mathrm{x} 2$ in the first equation, that $\mathrm{x} 1=$ $1 / 3$.

Systems of linear equations

\subsection{Linear equations}

Before going on, let us reformulate the notion of a system of linear equations into the language of functions. This will also help us understand the adjective Vinear" a bit better. A function $\mathrm{f}$ is a map

$$
f: X \rightarrow Y
$$

From a set $\mathrm{X}$ to a set $\mathrm{Y}$. The set $\mathrm{X}$ is called the domain of the function, and the set $\mathrm{Y}$ is called the target space or codomain of the function. An equation is

$$
f(x)=y,
$$

A line going through the start (blue, thick) in $\mathrm{R}$ is a straight subspace, a not unpredictable thing of explore direct factor based math. Direct factor based math is a division of science required with the preliminary of vectors, vector domains (besides implied as straight spaces), straight maps (moreover known as straight changes), and structures of direct conditions. Vector zones are a principle subject in cutting edge math; subsequently, straight factor based math is generally utilized in every rundown polynomial math and useful appraisal. Straight polynomial math moreover has a strong depiction in analytical geometry and it's miles summed up in chairman thought. It has critical packages inside the home developed sciences and the humanistic systems, on account of reality nonlinear styles can much of the time be approximated by using direct ones.

\subsection{Mathematical Statements}

In a logical confirmation or talk one makes severa verifications, reliably called clarifications or sentences.1For example:

\section{1. $(x+y)^{2}=x^{2}+2 x y+y^{2}$.}

2. $3 x^{2}+2 x-1=0$.

\section{3. if $n(\geq 3)$ is an integer then $a^{n}+b^{n}=c^{n}$ has no positive integer solutions.}

\section{4. the derivative of the function $x^{2}$ is $2 x$.}

Although a mathematical statement always has a very precise meaning, certain things are often assumed from the context in which the statement is made. For example, depending on the context in which statement (1) is made, it is probably an abbreviation for the statement for all real

numbers $x$ and $y,(x+y)^{2}=x^{2}+2 x y+y^{2}$.

However, it may also be an abbreviation for the statementfor all complex numbers $x$ and $y$, $(x+y)^{2}=x^{2}+2 x y+y^{2}$.

The precise meaning should always be clear from context; if it is not then more information should be provided.

Statement (2) probably refers to a particular real number $x$; although it is possibly an abbreviation for the (false) statementfor all real numbers $x$, $3 x^{2}+2 x-1=0$.

\section{THE REAL NUMBER SYSTEM}

\subsection{Introduction}

The real quantity machine satisfies certain axioms, from which its distinctive houses can be deduced. There are numerous slightly exclusive, but equal, formulations.

Definition 3.1.1 The real big variety machine is a set 1 of things known as real Numbers and implied with the resource of $\mathrm{R}$ collectively with parallel operations 2 known as extension and increase and proven by using manner of the use of + and $*$ independently (we generally create $\mathrm{xy}$ for $\mathrm{x} *$ $y)$, a twofold association known as no longer genuinely and implied with the beneficial useful resource of $<$, and indisputable segments called zero and harmony and implied through zero and 1 independently.

The maxims glad through the use of approach of those fall into 3 social activities and are component through way of the usage of the usage of element within the going with territories.

\subsection{Algebraic Axioms}

Numerical homes are the homes of the 4 physical sports sports: extension + , duplication *, subtraction $i$, and department $¥$. 
Houses of Addition If $\mathrm{a}, \mathrm{b}$ and $\mathrm{c}$ are real numbers with the useful resource of then:

A1 $a+b=b+a$

$\mathrm{A} 2(\mathrm{a}+\mathrm{b})+\mathrm{c}=\mathrm{a}+(\mathrm{b}+\mathrm{c})$

A3 a+zero $=0+a=a$

A4 there is really one real huge range, tested by way of $i a$, with the actual aim that $a+(j a)=(j a)+a=$ zero assets A1 is referred to as the commutative property of extension; it says it does now not have any shape of effect how one drives (trades) the solicitation of improvement. Property A2 says that if we encompass an and $b$, and a while later add $c$ to the surrender end end result, we get proportional to which incorporates a to the not on time end result of collectively with $b$ and c. It's far referred to as the agreeable assets of improvement; it would now not have any sort of effect how we accomplice (be a part of) the areas. The like final effects isn't always valid for subtraction or branch.

Assets A3 says there can be a super veritable variety zero, known as 0 or the more substance person, which while delivered to any actual quantity a, offers a. Belongings A4 says that for any certifiable widespread variety a there can be an unusual (as an example decisively one) veritable variety $i a$, called the terrible or blanketed substance in opposite of a, which at the same time as delivered to a offers 0 .

Homes of Multiplication If $\mathrm{a}, \mathrm{b}$ and $\mathrm{c}$ are certified numbers through then:

A5 $\mathrm{a} * \mathrm{~b}=\mathrm{b} * \mathrm{a}$

$\mathrm{A} 6(\mathrm{a} * \mathrm{~b}) * \mathrm{c}=\mathrm{a} *(\mathrm{~b} * \mathrm{c})$

A7 $\mathrm{a} * 1=1 * \mathrm{a}=\mathrm{a}$, and $16=$ zero.

A8 if a $6=0$ there's simply one certified large range, established thru $a_{i} 1$, with the authentic aim that

$$
\mathrm{A} * \mathrm{a}_{i} 1=\mathrm{a}_{i} 1 * \mathrm{a}=1
$$

Homes A5 and A6 are called the commutative and acquainted houses for duplication.

Belongings A7 says there can be an authorized number one $6=$ zero, known as one or the multiplicative individual, which on the equal time as copied through any actual range a, offers a.

Property A8 says that for any non-0 veritable amount a there can be a completely unique certifiable range $a_{j} 1$, known as the multiplicative in contrary of a, which on the equal time as copied via a offers 1 .

Display we are able to as often as viable form stomach muscle for $\mathrm{a} * \mathrm{~b}$.

The Distributive property there's each different belongings which incorporates every extension and growth:

A9 If $a, b$ and $c$ are veritable numbers with the useful aid of then

$$
\mathrm{A}(\mathrm{b}+\mathrm{c})=\text { stomach muscle }+ \text { cooling }
$$

The distributive property says that we will autonomously suitable increment over the 2 included substance phrases

Numerical Axioms it would appear you could nonetheless show all the logarithmic homes

Of the real numbers from houses A1A9 of extension and growth. We're in a role to finish a section of this inside the accompanying subsection. We name A1A9 the Algebraic Axioms for the certified huge variety device.

Consistency you could in reality file various homes of correspondence. Specially, for each veritable extensive range $\mathrm{a}, \mathrm{b}$ and $\mathrm{c}$ :

1. $A=a$

2. $A=b) b=a 3$

3. $A=b$ and $4 b=c) a=c 5$

Moreover, in case $\mathrm{a}=\mathrm{b}$, via then $\mathrm{a}+\mathrm{c}=\mathrm{b}+\mathrm{c}$ and cooling $=$ bc. Even more all subjects considered, viable for the maximum trouble update a time period in an enunciation via some special term to which it is similar.

It's far possible to file sayings for $="$ and come to be diverse houses of $="$ from the ones adages; yet we do no longer do that. Or in all likelihood, we take =" to be a savvy idea this indicates that may be a comparative detail as"; the beyond houses of $=$ " are then valid from the importance of $="$. While we make a $6=\mathrm{b}$ we will advocate that a does not deal with a comparable variety as $b$; as an instance an addresses a di®erent full-size variety from $b$.

Considered one of a kind Logical and Set Theoretic Notions We do not try to axiomatise any of the eager musings related to tremendous variety-crunching, nor do we try to axiomatise any of the homes of units which we are able to use (see later). It's far viable to do this; and this turns on a few uncommonly big and massive results concerning the man or woman and foundations of era. See later instructions at the foundations range-crunching (additionally more than one courses inside the hypothesis place of job).

\subsubsection{Results of the Arithmetical Proverbs}

Subtraction and department We in the beginning describe subtraction to the quantity improvement and the additional substance communicate, thru the use of

Manner of

$$
a-b=a+(-b) \text {. }
$$

$$
a \div b\left(=a / b=\frac{a}{b}\right)=a b^{-1} .
$$

A couple of aftereffects of adages A1A9 are according to the accompanying. The affirmations are given inside the AH1 notes.

Theory 3.2.1 (Cancellation law for Addition) If a, b and c are certifiable numbers and $\mathrm{a}+\mathrm{c}=\mathrm{b}+\mathrm{c}$, by then $\mathrm{a}=\mathrm{b}$.

Theory 3.2.2 (Cancellation law for Multiplication) If $a, b$ and c $6=$ zero are certifiable numbers and cooling $=b c$ then $a$ $=\mathrm{b}$.

Theory 3.2.three If $a, b, c, d$ are certifiable numbers and c $6=0, \mathrm{~d} 6=0$ by then
1. $\mathrm{A} 0=0$
2. $i(j a)=a$
3. $\left(c_{i} 1\right) ; 1=c$
4. $(; 1) a=i a$
5. $A(; b)=i(a b)=(; a) b$
6. $(j a)+(j b)=i(a+b)$
7. $(; a)(; b)=$ muscular strength
8. $(a=c)(b=d)=(a b)=(c d)$
9. $(a=c)+(b=d)=($ advert $+b c)=c d$

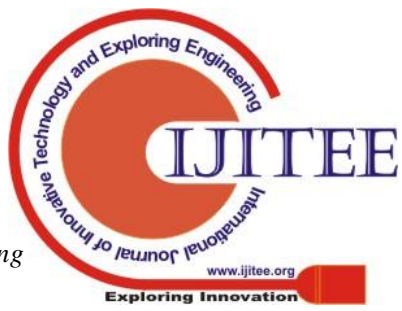


Presentation Henceforth (beside we are expressing in some other case) we will depend on all the standard spots of development, growth, subtraction and office. remarkably, we can resolve synchronous straight conditions. we're ready to do other than acknowledge favored definitions which fuse $\mathrm{x} 2=\mathrm{x} * \mathrm{x}, \mathrm{x} 3=\mathrm{x} * \mathrm{x} * \mathrm{x}, \mathrm{x}_{i} 2=\left(\mathrm{x}_{i} 1\right) 2$, and so on.

\subsubsection{Crucial Courses of Action of Real Numbers}

We chart

$$
\begin{gathered}
2=1+1 ; \text { three }=2+1 ;: ; 9=8+1 ; \\
10=9+1 ;: ; 19=18+1 ;: ; 100=99+1 ;:
\end{gathered}
$$

The set $\mathrm{N}$ of trademark numbers is portrayed by strategy for method for $\mathbf{N}=\{1,2,3, \ldots\}$.

The set $\mathbf{Z}$ of integers is defined by

$$
\mathrm{Z}=\{m:-m \in \mathrm{N} \text {, or } m=0 \text {, or } m \in \mathrm{N}\} \text {. }
$$

The set $\mathbf{Q}$ of rational numbers is defined by

$$
\mathbf{Q}=\{m / n: m \in \mathbf{Z}, n \in \mathbf{N}\} \text {. }
$$

The set of all real numbers is denoted by $\mathbf{R}$.

A real number is irrational if it is not rational.

\subsubsection{The Order Axioms}

As remarked in Section 3.1, the real numbers have a natural ordering. Instead of writing down axioms directly for this ordering, it is more convenient to write out some axioms for the set $P$ of positive real numbers. We then define <in terms of $P$.

Order Axioms There is a subset6 $P$ of the set of real numbers, called the set of positive numbers, such that:

A10 For any real number $a$, exactly one of the following holds:

$$
a=0 \text { or } a \in P \text { or }-a \in P
$$

\section{A11 If}

$$
a \in P \text { and } b \in P \text { then } a+b \in P \text { and } a b \in P
$$

A number ais called negative when - $a$ is positive.

\section{SET THEORY\& RESULTS}

\subsection{Introduction}

The possibility of a hard and speedy is vital to math. A difficult and fast is, coolly, a assembly of articles. We can't use this as a definition anyways, as we through then need to explain what we advise with the beneficial useful resource of a fixed. The possibility of a difficult and rapid is a easy or unrefined one, as is enlistment 2, which are not typically portrayed with appreciate to severa thoughts. same phrases for set are accumulating, class1 and family.

It is viable to file platitudes for the idea of devices. To try this fittingly, one furthermore wants to formalize the technique of reasoning included. We won't are searching out for after this kind of famous technique to control set idea, however will alternatively preserve in a dynamically easygoing way.

Gadgets are big as it is feasible to layout all of math in set speculation. This is not finished in advance than prolonged, in any case, other than at the off risk that one is obsessed with the hints of Mathematics2.

\subsection{Russell's Paradox}

It would have all the earmarks of being affordable to renowned that for any belongings" or scenario" $\mathrm{P}$, there may be a hard and rapid $S$ containing all things with the given property. Even extra effectively, if $\mathrm{P}(\mathrm{x})$ indicates that $\mathrm{x}$ has belongings $\mathrm{P}$, through way of way of then there mustBe a

fixed $S$ defined via $S=\{x: P(x)\}$.

This is read as: $W$ is the set of all $x$ such that $P(x)$ (is true)"3. For example, if $P(x)$ is an abbreviation for $x$ is an integer >5or $x$ is a pink elephant; then there is a corresponding set (although in the second case it is the so called empty set, which has no members) of objects $x$ having property $P(x)$.

$$
x \notin x \text {. }
$$

Suppose

$$
S=\{x: x \notin x\}
$$

In light of this present there can be a sensible irregularity in either case.

On the same time as this will show up a phony model, there emerges the pivotal bother of comprehending which houses we should permit with a true aim to depict devices. This burden is considered inside the angle on worldwide renowned set idea. We are prepared to now not (preferably!) be the usage of homes that bring about such troubles, our age inside the above condition will however be of the shape given a fixed $\mathrm{A}$, endure in thoughts the segments of a top notch some portraying affects".

None-the-drastically much less, at the equal time as the German realist and mathematician GottlobFrege were given observe from Bertrand Russell (over the flip of the century) of the above property, comparably as the second one version of his sum paintings Grundgesetze der Arithmetik (The fundamental proper suggestions of range juggling) create as in press, he felt obliged to contain the following declaration:

An analyst can barely ever experience a few thing greater unwanted than to have the foundation breakdown basically in view of the fact crafted by using manner of expertise is finished. I was organized in this trademark thru a letter from Mr. Bertrand Russell while the canvases modified into nearly via the snap.

Give up Numerous variable based totally math situations may be anything apart from hard to determine on the equal time as others are more and more conspicuous confused and tough. It's miles critical to entice an technique to recuperation arithmetical conditions and use an outlining small scale pc to check your solutions as a result of the truth the direction intertwines over on your destiny math guidance, for instance, geometry, polynomial math 2 , and research. Inside the wake of finishing this agency the entirety about should display off sufficient self 
acknowledgment in solving arithmetical conditions with a actual aim to be prepared for assist different individuals who technique you for help.

\section{REFERENCES}

1. Ralph Abraham and Jerrold E. Marsden. Foundations of Mechanics. Addison Wesley, 2nd shape, 1978.

2. Lars V. Ahlfors and Leo Sario. Riemann Surfaces. Princeton Math. Path of motion, No. 2. Princeton college Press, 1960.

3. George E. Andrews, Richard Askey, and Ranjan Roy. Uncommon capabilities. Cambridge college Press, first shape, 2000.

4. Tom Apostol. Exam. Addison Wesley, 2nd variant, 1974.

5. V.I. Arnold. Logical structures for Classical Mechanics. GTM No. 102. Springer Verlag, second structure, 1989.

6. Emil Artin. Geometric Algebra. Wiley Interscience, first form, 1957.

7. Michael Artin. Polynomial math. Prentice lobby, first form, 1991.

8. M. F. Atiyah and i. G. Macdonald. Coming to Commutative Algebra. Addison Wesley, zero.33 adjustment, 1969.

9. A. Avez. CalculDiff'erentiel. Masson, first shape, 1991.

10. Sheldon Axler. Instantly Algebra completed proper. Scholar Texts in variety juggling. Springer Verlag, second version, 2004. 